\title{
Lente fácica de câmara posterior para correção de hipermetropia consecutiva à ceratotomia radial
}

\author{
Posteriorchamber phakiclens for thecorrection of hyperopia following radial keratotomy
}

\author{
Cristina Moreira Salera ${ }^{1}$ \\ Edgar Emiliano Duarte Servian ${ }^{1}$ \\ Fernando Bonfim Moreira ${ }^{1}$ \\ Márcia Reis Guimarães ${ }^{2}$ \\ Raul Damásio Castro ${ }^{3}$ \\ Ricardo Queiroz Guimarães ${ }^{4}$
}

\begin{tabular}{l} 
RESUMO \\
\hline Objetivo: Avaliar a eficácia, previsibilidade e segurança do implante de \\
lente fácica de câmara posterior para a correção de hipermetropia conse- \\
cutiva à ceratotomia radial. Métodos: Foram analisados retrospectivamen- \\
te os prontuários de seis pacientes (10 olhos) com hipermetropia conse- \\
cutiva à hipercorreção após ceratotomia radial submetidos ao implante de \\
lente fácica de câmara posterior para sua correção. Resultados: O equiva- \\
lente esférico pré-operatório médio da refração dinâmica foi de $+4,65$ \\
dioptrias (variando de $+2,50$ a $+6,50$ dioptrias) e o equivalente esférico pós- \\
operatório médio foi de $+0,3375$ dioptrias (variando de $-0,875$ a $+2,25$ \\
dioptrias). Quando comparamos as acuidades visuais com correção pré e \\
pós-operatórias observamos que três olhos (30\%) ganharam duas linhas \\
de acuidade visual, dois (20\%) ganharam uma linha de acuidade visual, um \\
(10\%) manteve a mesma acuidade visual, três olhos (30\%) perderam uma \\
linha de acuidade visual e um olho (10\%) perdeu duas linhas. Conclusão: \\
Oimplante da lente fácica de câmaraposterior para correção da hipermetropia \\
consecutiva à ceratotomia radial mostrou-se como uma nova opção para \\
otratamento desta indesejável complicação, entretanto, fazem-se necessá- \\
rios estudos abrangendo maior número de casos e um acompanhamento \\
a longo prazo destes casos.
\end{tabular}

Descritores: Lentes intra-oculares; Implante de lente intra-ocular; Hiperopia/cirurgia; Hiperopia/etiologia; Refração ocular; Ceratotomia radial/efeitos adversos
Este trabalho foi realizado no Hospital de Olhos de Minas Gerais - Clínica Dr. Ricardo Guimarães.

Fellow em Clínica e Cirurgia do Segmento Anterior do Hospital de Olhos de Minas Gerais.

2 Doutora em Oftalmologia pela Universidade Federal de

Minas Gerais, Patologista Ocular e Diretora do Hospital de Olhos de Minas Gerais.

${ }^{3}$ Cirurgião da Unidade de Cirurgia Refrativa do Hospital de Olhos de Minas Gerais.

${ }^{4}$ Doutor em Oftalmologia pela Universidade Federal de Minas Gerais, Cirurgião-chefe e Diretor do Hospital de Olhos de Minas Gerais.

Endereço para correspondência: Cristina Moreira Salera, Rua da Paisagem, 222 Belo Horizonte (MG) CEP 30161-970 E-mail: csalera@uai.com.br

Recebido para publicação em 26.09.2002 Aceito para publicação em 06.03.2003

\section{INTRODUÇ̃̃̃O}

A ceratotomia radial foi introduzida nos Estados Unidos em 1979 e foi amplamente utilizada para a correção da miopia baixa a moderada ${ }^{(1-2)}$. Entretanto, existem registros comprovando a tentativa de alteração cirúrgica da curvatura corneana ainda no século XIX.

A hipercorreção é uma das suas possíveis complicações cujos tratamentos têm se mostrado insatisfatórios De acordo com o estudo PERK (Prospective Evaluation of Radial Keratotomy) a hipermetropia progressiva foi relatada em $43 \%$ dos olhos entre 6 meses e 10 anos após a realização da ceratotomia radial $^{(2-3)}$ e as razões para este fenômeno ainda não foram definidas $^{(3)}$. As possíveis causas podem estar relacionadas a um planejamento cirúrgico inadequado ou a uma resposta cicatricial alterada além de fatores relacionados à técnica cirúrgica.

A lente intra-ocular fácica de câmara posterior (ICL) (Staar Surgical AG) é uma lente intrao-cular fácica de câmara posterior que deve ser posicionada entre a superfície posterior da íris e a superfície anterior do cristalino ${ }^{(4)}$.

As vantagens desta modalidade de cirurgia refrativa incluem a sua reversibilidade, a preservação da acomodação e a não dependência de fatores cicatriciais da córnea ${ }^{(4-8)}$. 
Neste trabalho descrevemos uma série de pacientes que apresentaram hipermetropia após a ceratotomia radial e que foram tratados com o implante de lente fácica de câmara posterior.

\section{MÉTODOS}

Foram analisados, retrospectivamente, os dados de dez olhos de seis pacientes que procuraram o Hospital de Olhos de Minas Gerais com queixa de baixa acuidade visual após a realização de ceratotomia radial para correção de miopia com média de pós-operatório de oito anos (variando de 2 a 16 anos).

As cirurgias para implante da ICL ocorreram no período de junho de 1997 a novembro de 2000 e foram realizadas pelo mesmo cirurgião.

Este trabalho faz parte de um protocolo institucional de pesquisa do Hospital de Olhos de Minas Gerais de caráter experimental e aprovado por uma comissão de ética médica interna. Os pacientes foram informados sobre o caráter experimental do procedimento, riscos e benefícios e concordaram em assinar o termo de consentimento.

A avaliação pré-operatória incluiu os seguintes exames:

- refração dinâmica e estática;

- medida da pressão intra-ocular;

- medida da profundidade da câmara anterior (ecógrafo A com sonda de contato);

- medida da espessura da córnea (paquímetro ultra-sônico);

- medida do diâmetro corneano (compasso);

- topografia corneana computadorizada de elevação;

- biomicroscopia;

- fundoscopia;

- microscopia especular (microscópio especular computadorizado sem contato).

O objetivo refracional da cirurgia era o equivalente esférico mais próximo à emetropia) (ICL corrige somente o componente esférico da ametropia).

Com o objetivo de evitar o bloqueio pupilar pós-operatório, foram realizadas duas iridotomias com uma distância de $90^{\circ}$ uma da outra através dos lasers argônio e YAG, pelo menos uma semana antes da cirurgia.

A rotina para a preparação do paciente consistiu em sedação com midazolan (Dormonid ${ }^{\circledR}$ ) $3 \mathrm{ml}$ intravenoso e cloridrato de fentanil 0,5 mg $\left(\right.$ Fentanil $\left.^{\circledR}\right) 1 \mathrm{ml}$ intravenoso. Para dilatação da pupila utilizou-se uma gota dos seguintes colírios: cloridrato de proximetacaína (Anestalcon ${ }^{\circledR}$ ), tropicamida $\left(\right.$ Mydriacyl $\left.^{\circledR}\right)$, cloridrato de fenilefrina $\left(\right.$ Fenilefrina $\left.{ }^{\circledR}\right)$ e suprofeno (Procofen ${ }^{\circledR}$ ). Em um paciente (dois olhos) foi realizada a anestesia tópica com cloridrato de bupivacaína $0,75 \%$ sem vasoconstritor, 20 gotas em 15 minutos. Nos outros cinco pacientes (oito olhos) realizou-se o bloqueio peribulbar utilizando $6 \mathrm{ml}$ de cloridrato de lidocaína $2 \%$ com vasoconstritor (1:400.000).

Quanto à técnica cirúrgica, inicialmente duas incisões auxiliares eram realizadas afastadas $180^{\circ}$ uma da outra e a $90^{\circ}$ da incisão principal que era realizada no lado temporal ou no meridiano mais curvo. A extensão padrão da incisão principal era 3,0 mm podendo ser estendida para 3,2 a 3,5 $\mathrm{mm}$. A seguir, foi injetada substância viscoelástica de baixo peso molecular $\left(\right.$ Viscoat $\left.^{\circledR}\right)$ dentro da câmara anterior. A ICL era removida do frasco e colocada no injetor fornecido pelo fabricante. Uma vez a ICL implantada na câmara anterior, as suas extremidades distal e, posteriormente, a proximal, eram cuidadosamente posicionadas atrás da íris. Em seguida, a espátula de Guimarães (ICL Manipulator, Katena Inc.) foi usada para a colocação da lente sob a íris. Um mantenedor de câmara anterior com irrigação contínua de BSS era posicionado em uma das incisões auxiliares e a substância viscoelástica era removida da câmara anterior e do espaço entre a lente e o cristalino com o sistema de irrigação/aspiração. Ao final do procedimento provocavase a miose com a injeção na câmara anterior de carbachol $\left(\right.$ Miostat $^{\circledR}$ ) 0,01\%,1 ml diluído 1:1. Foi utilizada sutura para fechamento da incisão apenas naqueles casos cuja incisão apresentou extensão superior a 3,2 $\mathrm{mm}$. O paciente era examinado na lâmpada de fenda algumas horas após a cirurgia para assegurar que a câmara anterior estivesse com a profundidade adequada, a lente bem posicionada e não houvesse resíduos de viscoelástico. Foi prescrito para uso tópico a associação antibiótico/corticóide (sulfato de neomicina/sulfato de polimixina B e dexametasona) inicialmente quatro vezes ao dia com redução de uma gota a cada semana. Na avaliação pós-operatória foram realizadas: refração dinâmica, medida da pressão ocular, biomicroscopia e fundoscopia.

\section{RESULTADOS}

O grupo estudado era composto por 10 olhos de seis pacientes sendo que dois $(33,3 \%)$ pacientes eram do sexo feminino e quatro $(66,7 \%)$ do sexo masculino. Destes pacientes, quatro tiveram a ICL implantada em ambos os olhos e dois somente no olho esquerdo. A idade variou de 35 a 49 anos com média igual há 41,4 anos. $\mathrm{O}$ intervalo de tempo médio entre a realização da ceratotomia radial e o implante da ICL foi de 8,6 anos (variando de 2 a 16 anos).

A média do equivalente esférico obtido na refração dinâmica pré-operatória foi de $+4,65$ dioptrias, variando de $+2,50$ a $+6,50$ dioptrias. A média do equivalente esférico obtido na refração estática pré-operatória era de $+5,5$ dioptrias variando de $+3,25$ a $+6,75$ dioptrias. A ametropia cilíndrica média pré-operatória era de $-1,55$ dioptrias variando de 0 a $-3,00$ dioptrias. Após o implante da ICL, no primeiro dia de pós-operatório a média do equivalente esférico na refração dinâmica foi de $+0,775$ dioptrias, variando de $-0,75$ a $+1,875$ dioptrias. No trigésimo dia de pós-operatório a média do equivalente esférico na refração dinâmica foi de $+0,3375$ dioptrias, variando de $-0,875 \mathrm{a}+2,25$ dioptrias. A tabela 1 mostra os principais dados dos olhos analisados.

A ametropia cilíndrica pós-operatória teve média de -1,825 dioptrias (variando de $0 \mathrm{a}-4,00 \mathrm{D}$ ).

Quando comparamos a acuidade visual com correção préoperatória e a acuidade visual com correção pós-operatória observamos que três olhos (30\%) ganharam duas linhas de visão, dois olhos (20\%) ganharam uma linha de acuidade vi- 
sual, um olho (10\%) manteve a mesma acuidade visual, três olhos (30\%) perderam uma linha e um olho (10\%) perdeu duas linhas de acuidade visual. O gráfico 1 mostra que a acuidade visual corrigida pós-operatória foi $20 / 20$ em quatro olhos (40\%) enquanto que no pré-operatório somente dois olhos (20\%) apresentavam esta visão. O gráfico também mostra que um olho (10\%) apresentou visão $20 / 40$ no pós-operatório, visão que não foi observada no pré-operatório.

Ao compararmos a acuidade visual corrigida pré-operatória com a acuidade visual sem correção pós-operatória notamos que $20 \%$ dos casos ganharam uma linha de acuidade visual, $10 \%$ mantiveram a mesma acuidade visual, $40 \%$ perderam uma linha, $10 \%$ perderam duas linhas e $20 \%$ perderam mais que três linhas de acuidade visual.

Destes 10 olhos, cinco ainda se submeteram a outros procedimentos com o objetivo de alcançar melhor acuidade visual: em um olho foi realizada a sutura circular (caso 1), em dois olhos fotoablação com a técnica LASIK (casos 3 e 4), em função da hipocorreção do componente esférico (hipermetropia). Em dois casos ( 7 e 8) foi indicado o uso de lentes de contato rígidas devido ao alto astigmatismo que se manteve após a cirurgia. $\mathrm{O}$ seguimento dos pacientes foi em média de 41 meses (variando de 5 a 65 meses). Com exceção dos olhos que necessitaram de outro procedimento refrativo cirúrgico (casos 1,3 e 4), observamos um equivalente esférico médio de $+0,14$ dioptrias (variando de $-0,75 \mathrm{a}+1,00 \mathrm{D})$ durante $\mathrm{o}$ acompanhamento.

$\mathrm{E}$, finalmente, três anos após o implante da lente fácica, dois olhos de um mesmo paciente (casos 9 e 10) foram submetidos à facoemulsificação com implante de lente intra-ocular devido à formação de opacificação do cristalino e visão menor que 20/70 sem melhora com a refração.

\section{DISCUSSÃO}

A hipermetropia conseqüente à ceratotomia radial é uma complicação freqüente e indesejável após a ceratotomia radial ${ }^{(1)}$ que pode-se dever à fragilidade causada à estrutura da córnea através das incisões com aplanamento progressivo da área central.

Além do PERK, outros estudos mostraram um padrão se-

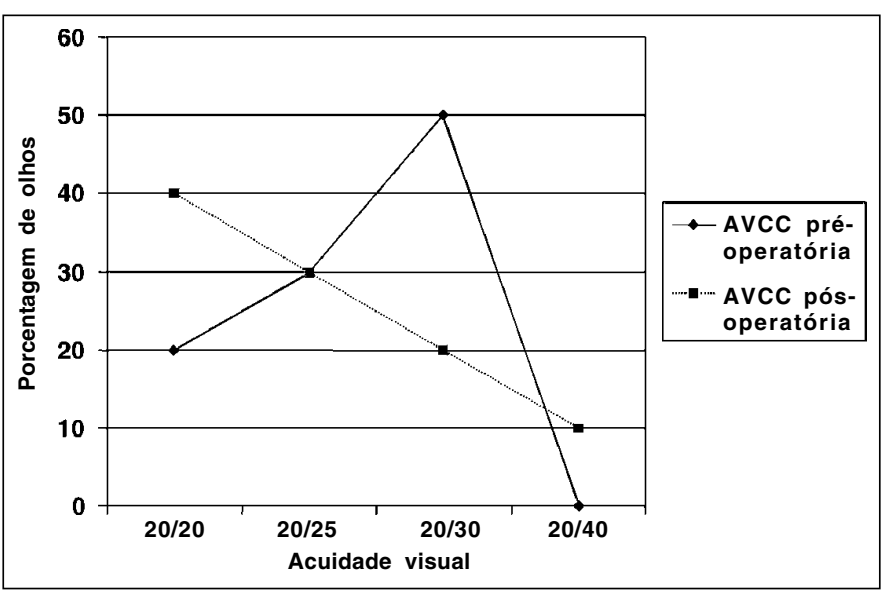

Gráfico 1 - Comparação das acuidades visuais com correção pré e pós-operatórias

melhante com uma incidência de aproximadamente $30 \%$ de hipermetropia progressiva entre um e quatro anos após a cirurgia. As possíveis causas para a hipercorreção envolvem uma avaliação pré-operatória inadequada, incisões radiais muito amplas estendendo-se até o limbo, várias reoperações (novas incisões), procedimentos para aprofundar as incisões, uso prolongado de lentes de contato após a ceratotomia radial e o hábito de esfregar os olhos ${ }^{(1)}$.

A manipulação farmacológica da acomodação com o uso do colírio de cloridrato de pilocarpina tem sido utilizada para controlar graus leves de hipercorreção, mas o seu efeito é pouco previsível e temporário ${ }^{(9)}$.

O momento ideal para a tentativa de correção da hipermetropia conseqüente à ceratotomia radial nos parece ser aquele em que observamos uma estabilidade refracional.

Alguns procedimentos foram utilizados como tentativa de tratar a hipercorreção como sutura circular, sutura das incisões com pontos separados, ceratoplastia por rádio freqüência e ceratoplastia lamelar, mas não mostraram resultados satisfatórios ${ }^{(1,7,9-10)}$. A ceratectomia fotorrefrativa com excimer laser (PRK, do inglês, photorefractive keratectomy) mostrou um risco aumentado de ocorrência de "haze" e conseqüente

\begin{tabular}{|c|c|c|c|c|c|c|c|c|c|}
\hline Caso & Idade & Sexo & Tempo de RK (anos) & Modelo ICL & Refração pré & AVCC pré & AVSC pós & Refração pós & AVCC pós \\
\hline 1 & 39 & $\mathrm{~F}$ & 4 & 120V2 & $+4,50-4,00 \times 120$ & $20 / 25$ & $20 / 80$ & $+4,25-4,00 \times 120$ & $20 / 25$ \\
\hline 2 & 39 & M & 12 & $115 \mathrm{~V} 3$ & $+5,00-1,00 \times 95$ & $20 / 30$ & $20 / 40$ & $-0,25-0,75 \times 85$ & $20 / 25$ \\
\hline 3 & 49 & M & 16 & $120 \mathrm{~V} 3$ & $+7,00-3,00 \times 60$ & $20 / 25$ & $20 / 150$ & $+3,00-4,00 \times 55$ & $20 / 20$ \\
\hline 4 & 49 & M & 16 & $120 \mathrm{~V} 3$ & $+6,25-1,25 \times 105$ & $20 / 30$ & $20 / 30$ & $+3,00-2,00 \times 105$ & $20 / 20$ \\
\hline 5 & 35 & M & 7 & $120 \mathrm{~V} 3$ & $+5,00-2,00 \times 160$ & $20 / 30$ & $20 / 25$ & $+0,50-1,00 \times 115$ & $20 / 20$ \\
\hline 6 & 35 & M & 7 & $120 V 3$ & $+4,00-1,75 \times 80$ & $20 / 30$ & $20 / 20$ & $+1,00-1,50 \times 105$ & $20 / 20$ \\
\hline 7 & 37 & M & 2 & $115 \mathrm{~V} 3$ & $+7,00-3,00 \times 100$ & $20 / 30$ & $20 / 40$ & $+1,00-3,75 \times 140$ & $20 / 40$ \\
\hline 8 & 37 & M & 2 & $115 \mathrm{~V} 3$ & $+6,50-1,50 \times 90$ & $20 / 20$ & $20 / 30$ & Plano-1,25x90 & $20 / 30$ \\
\hline 9 & 47 & $\mathrm{~F}$ & 10 & $115 \mathrm{~V} 3$ & $+7,50-2,00 \times 80$ & $20 / 20$ & $20 / 25$ & Plano & $20 / 25$ \\
\hline 10 & 47 & $\mathrm{~F}$ & 10 & $115 \mathrm{~V} 3$ & $+4,00-0,75 \times 45$ & $20 / 25$ & $20 / 30$ & Plano & $20 / 30$ \\
\hline
\end{tabular}


redução da acuidade visual ${ }^{(1-2,9)}$. A técnica ceratectomia lamelar fotorrefrativa (LASIK, do inglês, laser-assisted in situ keratomileusis) também tem sido utilizado e tem mostrado resultados encorajadores embora devamos sempre ter em mente o risco aumentado para complicações como ressecção lamelar irregular, as limitações de espessura e topografia corneanas, abertura das incisões ${ }^{(1-2)}$, formação de crescimento epitelial e ocorrência de astigmatismo irregular ${ }^{(9)}$.

Por outro lado, a previsibilidade do implante da lente fácica de câmara posterior também tem limitações. Esta técnica depende da acurácia da biometria e da ceratometria que pode ser prejudicada após uma cirurgia corneana devido às alterações topográficas da córnea ${ }^{(7)}$.

Outra limitação é a indução de um aplanamento corneano adicional pelo implante de uma lente intra-ocular em olhos que foram submetidos à ceratotomia radial(7).

As vantagens do implante de ICL para correção da hipermetropia após ceratotomia radial incluem a sua reversibilidade, o fato de a ICL ser uma lente intra-ocular dobrável que pode ser implantada através de uma incisão de $2,8 \mathrm{~mm}$ minimizando os riscos de alterações indesejáveis na curvatura corneana ${ }^{(7)}$. Além disso, existe a possibilidade de atenuar o astigmatismo através do correto posicionamento e extensão adequada da incisão para implante da $\mathrm{LIO}^{(7)}$. Apesar de todos os cuidados, observamos em alguns pacientes uma instabilidade das incisões corneanas da ceratotomia radial com um conseqüente aumento da ametropia cilíndrica, o que é relatado na literatura ${ }^{(7)}$.

No presente estudo, durante o seguimento (que foi em média de 41 meses) daqueles casos que não se submeteram a outro procedimento refrativo cirúrgico observamos uma estabilidade quanto à refração e à manutenção da acuidade visual. Também é importante ressaltar que um acompanhamento a longo prazo dos casos poderia ainda demonstrar uma falta de estabilidade da hipermetropia mesmo após o implante da ICL.

Quanto às complicações deste tipo de procedimento podemos encontrar: bloqueio pupilar pós-operatório, depósitos de pigmentos sobre a lente e catarata ${ }^{(5-6)}$. A opacificação do cristalino pode ocorrer devido a vários fatores como a proximidade entre a lente e o cristalino, trauma durante a implantação da lente, alterações do metabolismo lenticular causados pela presença da lente ou pelos restos de substância viscoelástica ${ }^{(5)}$.

\section{CONCLUSÃO}

O nosso estudo, apesar do número limitado de pacientes, mostrou uma nova opção para se tentar a correção da hipermetropia consecutiva à ceratotomia radial. Embora os resultados não sejam tão encorajadores quanto àqueles encontrados na correção da hipermetropia primária, o ganho de linhas de acuidade visual em cinco olhos (50\%) e a melhora refracional estimula a realização de estudos abrangendo um número maior de casos e um acompanhamento a longo prazo são para melhor avaliação da técnica.

ABSTRACT

Purpose: To evaluate the efficacy, predictability and safety of posterior chamber phakic intraocular lens implantation in patients with hyperopia following radial keratotomy. Methods: Retrospective analysis of six patients (10 eyes) previously submitted to radial keratotomy, who presented consecutive hyperopia and were treated with implantation of a posterior chamber phakic intraocular lens. Results: The mean preoperative spherical equivalent manifest refraction was $+4.65 \mathrm{D}$ (ranging from +2.50 to $+6.50 \mathrm{D}$ ) and the mean postoperative spherical equivalent obtained with manifest refraction was $+0.3375 \mathrm{D}$ (ranging from -0.875 to $+2.25 \mathrm{D}$ ). Comparing the preoperative and postoperative corrected visual acuity, we observed that three eyes (30\%) gained two lines of visual acuity, two (20\%) gained one line, one $(10 \%)$ kept the same visual acuity, three eyes (30\%) lost one line of visual acuity and one eye (10\%) lost two lines. Conclusion: The implantation of posterior chamber phakic lens in order to correct hyperopia following to radial keratotomy is a new option of treatment for this undesirable complication but a larger number of eyes and a longer follow-up are required.

Keywords: Lenses, intraocular; Lens implantation, intraocular; Hyperopia/cirurgia; Hyperopia/etiology; Refraction, ocular; Keratotomy, radial/adverse effects

\section{REFERENCIAS}

1. Attia VH, Alió JL, Artola A, Muñoz G, Shalaby AM. Laser in situ keratomileusis for undercorrection and overcorrection after radial keratotomy. J Cataract Refract Surg 2001;27:267-72.

2. Lipshitz I, Man O, Shemesh G, Lazar M, Loewenstein A. Laser in situ keratomileusis to correct hyperopic shift after radial keratotomy. J Cataract Refract Surg 2001;27:273-6.

3. Waring GO III, Lynn MJ, McDonnell PJ. Results of the prospective evaluation of radial keratotomy (PERK) study 10 years after surgery [commented Arch Ophthalmol 1995;113:1225-6]. Arch Ophthalmol 1994;112:1298-308.

4. Visessook N, Peng Q, Apple D, Gere R, Schmicler S, Schoderbek RJ, et al. Pathological examination of a explanted phakic posterior intraocular lens. [commented on J Cataract Refract Surg 1999;25:1033-4]. J Cataract Refract Surg 1999;25:216-22.

5. Rosen E, Gore C. Staar Collamer posterior chamber intraocular lens to correct myopia and hyperopia. J Cataract Refract Surg 1998;24:596-606.

6. Davidorf JM, Zaldivar R, Oscherow S. Posterior chamber phakic intraocular lens for hyperopia of +4.00 to +11.00 diopters [commented on J Refract Surg 1998;14:272-3]. J Refract Surg 1998;14:406-11.

7. Fink AM, Gore C, Rosen ES. Overcorrected radial keratotomy treated with posterior chamber phakic intraocular lens and laser thermal keratoplasty. J Refractive Surg 1999;15:683-6.

8. Pershin KB, Pashinova NF. Refractive surgery for hyperopia. J Refract Surg 2000;16:242-6.

9. Francesconi CM, Nosé RA, Nosé W. Hyperopic laser-assisted in situ keratomileusis for radial keratotomy-induced hyperopia. Ophthalmology 2002;109: 602-5.

10. Lima Júnior RP, Neves RA, Nosé W, Campos MS. Avaliação clínica do tratamento cirúrgico da hipercorreção pós-ceratotomia radial. Arq Bras Oftalmol 1993;56:350-3. 